\title{
Retinal nerve fibre layer and ganglion cell complex thickness in patients with keratoconus
}

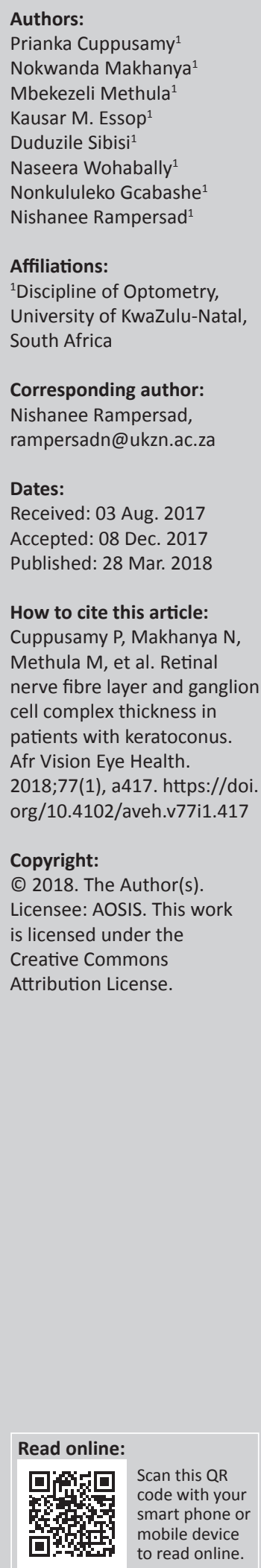

Background: Keratoconus, a corneal ectasia, is associated with corneal thinning and altered optical media. Consequently, assessment of the visual field, optic nerve head and intraocular pressure measurements may be challenging in patients with keratoconus. Few studies have investigated posterior segment variables including the retinal nerve fibre layer (RNFL) and ganglion cell complex (GCC) thickness in patients with keratoconus.

Aim: To investigate RNFL and GCC thickness in patients with keratoconus.

Methods: A comparative quantitative research design was used. The sample consisted of 56 participants ( 28 with mild, moderate or severe keratoconus, and 28 controls) who accessed the optometry clinic at the University of KwaZulu-Natal. There was an equal distribution of male $(n=14)$ and female $(n=14)$ participants in the keratoconus and control groups. Most participants were black $(n=34)$ or Indian $(n=18)$. Corneal power and refractive error were assessed with the Oculus Keratograph and subjective refraction respectively. The iVue-100 optical coherence topography device was used to measure RNFL and GCC thickness. Data were analysed by descriptive and inferential statistics.

Results: The mean global RNFL thickness was slightly higher in the control group than the keratoconus group for the right $(106 \mu \mathrm{m}$ vs. $99 \mu \mathrm{m})$ and left $(103 \mu \mathrm{m}$ vs. $98 \mu \mathrm{m})$ eyes but these differences were not significant $(p \geq 0.057)$. For all RNFL quadrants, slightly lower mean RNFL measurements were found in the keratoconus group. The mean GCC thicknesses were marginally higher $(3 \mu \mathrm{m}-6 \mu \mathrm{m})$ in the control group.

Conclusion: The RNFL and GCC thickness differences between patients with keratoconus and controls are not clinically significant. Therefore, abnormally reduced RNFL and GCC thickness measurements in patients with keratoconus warrant further investigation for other pathologies specifically glaucoma.

\section{Introduction}

Keratoconus is a bilateral asymmetrical non-inflammatory progressive corneal ectasia that affects one in every 2000 individuals in the general population. ${ }^{1}$ This ectasia is associated with a conical shaped cornea, apical protrusion and corneal thinning. ${ }^{2}$ Assessment of the visual field may be challenging in patients with keratoconus owing to corneal opacities, high refractive errors and/or higher order aberrations. ${ }^{3}$ Even though evaluation of optic disc photographs is a standard method to monitor optic nerve head changes in glaucoma suspects, this method may have limited usefulness in patients with keratoconus owing to poor-quality photographs and a relatively normal appearance of the optic nerve head. ${ }^{4}$ Moreover, patients with keratoconus usually present with myopia and low intraocular pressure (IOP) measurements. ${ }^{4}$ Even in the absence of keratoconus, IOP measurements may be underestimated in individuals with thinner corneas. ${ }^{1,5}$

Glaucoma is an optic neuropathy that may result in irreversible blindness. ${ }^{1}$ In a recent metaanalysis, Tham et al. ${ }^{6}$ estimated that the global prevalence of glaucoma will increase by $74 \%$ and affect approximately 112 million people by the year 2040. The assessment of IOP is used in the screening, diagnosing and monitoring of glaucoma, as IOP is a key modifiable factor linked to glaucoma. ${ }^{1,7}$ Since glaucoma is associated with myopia ${ }^{2}$ and the evaluation of IOP as well as visual fields may be unreliable in patients with keratoconus, assessment of the retinal nerve fibre layer (RNFL) may be more useful to screen and monitor glaucoma in patients with keratoconus. ${ }^{3}$ Moreover, the RNFL is part of the ganglion cell complex (GCC) which is sensitive to early glaucomatous changes. ${ }^{9}$ Various quantitative and qualitative methods may be used to assess the RNFLincluding fundus photography with a red-free filter, confocal scanning laser ophthalmoscopy, scanning laser polarimetry and optical coherence tomography (OCT). ${ }^{10}$ 
Optical coherence tomography, first introduced in $1991,{ }^{11}$ is an imaging technique that uses low-coherence interferometry to create high-resolution cross-sectional images (tomograms) of biological tissues of interest. ${ }^{10,11}$ The technique of OCT is similar to ultrasonography except that it is a non-contact technique and uses reflected light waves to create the tomograms. Currently, OCT devices are being widely used for imaging in several biomedical fields (cardiology, urology, respiratory medicine, dermatology and developmental biology) as well as for anterior and posterior ocular segment imaging. ${ }^{12,13,14,15}$ To this extent, several studies have reported on the practical use of OCT devices for ocular diseases associated with the retina, ${ }^{15,16}$ cornea, ${ }^{17,18}$ anterior chamber angle ${ }^{19,20}$ as well as glaucoma..$^{21,22}$ Moreover, studies have also reported that OCT devices are reliable for repeated measurements of RNFL thickness in individuals with keratoconus ${ }^{23,24}$ as well as individuals with normal corneas. ${ }^{25,26}$

The influence of keratoconus on anterior ocular segment structures is well known wherein several studies have compared anterior segment variables in patients with keratoconus and healthy controls. ${ }^{27,28,29}$ However, not much is known about the influence of keratoconus on posterior ocular segment structures. Therefore, this study was conducted to investigate posterior segment variables, including the RNFL and GCC thickness, in patients with keratoconus.

\section{Methodology}

The study employed a comparative quantitative research design and was conducted at the Westville campus of the University of KwaZulu-Natal (UKZN). The study population comprised of patients accessing the UKZN optometry clinic. Keratoconus (mild, moderate or severe) and control participants were recruited using convenience and purposive sampling respectively. The control participants were race, age and gender matched to the participants with keratoconus. Study participants were aged between 18 years and 33 years to minimise the effect of age on RNFL thickness, as previous studies $^{30,31}$ have reported a decrease in RNFL thickness with increasing age.

A complete ocular examination of both eyes, including case history, LogMAR distance visual acuity, refraction, slit lamp examination, ophthalmoscopy and IOP measurements, was conducted on all participants to assess their eligibility. Refractive error was determined by performing autorefraction which was subsequently refined with subjective refraction. Subjective refraction was used to determine the spherical equivalent by adding the sphere power to half the negative cylinder power. ${ }^{32}$ The Oculus Keratograph, which is a reliable topographer, ${ }^{33}$ was used to assess corneal power along the two principal meridians (K1 and K2). Participants with systemic and/or ocular diseases (other than keratoconus), history of ocular surgery and/or trauma, IOP $>21 \mathrm{mmHg}$, central corneal scarring and advanced keratoconus (K1 and/or K2 greater than $54 \mathrm{D}$ with corneal scarring) were excluded.

Participants were diagnosed with keratoconus based on corneal topography and the presence of clinical signs, associated with keratoconus, on slit lamp examination. The clinical signs of keratoconus included corneal thinning, Vogt striae, Fleischer's ring, corneal scarring and Munson's sign. The average corneal power along the two principal meridians (K1 and $\mathrm{K} 2$ ) were used to classify participants with keratoconus as either mild $(<48 \mathrm{D})$, moderate $(48 \mathrm{D}-54 \mathrm{D})$ or severe ( $>54$ D). Participants with average corneal power $>54 \mathrm{D}$ together with corneal scarring were classified as advanced keratoconus and excluded from the study.

The Optovue iVue-100 OCT device was used to scan and measure the RNFL and GCC thicknesses for both eyes of all participants. This Fourier-domain OCT device is capable of operating at 26000 A-scans per second with an axial resolution of $5 \mu \mathrm{m} \cdot{ }^{34}$ When scanning with the iVue-100 OCT device, participants were instructed to focus on the internal fixation target while the real-time image of the participant's eye and retina was monitored on the laptop screen. In accordance with the manufacturer's recommendations, ${ }^{34}$ repeat scans were taken when the scan was labelled as 'poor' on the laptop screen or had a scan quality index $<27$.

The RNFL thickness was assessed with the optic nerve head scanning protocol. This scanning protocol determines the global RNFL thickness and average thickness in the four quadrants (superior, inferior, nasal and temporal) along a $3.45 \mathrm{~mm}$ diameter circle when centred on the optic nerve head. The optic nerve head scanning protocol consists of 12 radial line scans of 459 A-scans each and 13 concentric rings of 429-969 A-scans centred on the optic nerve head. The optic nerve head scanning protocol displays the average global and quadrant RNFL thickness in an image display (Figure 1).

The GCC thickness was scanned and measured with the GCC scanning protocol, which consists of a single horizontal line scan and 15 vertical line scans. The horizontal and vertical line scans are $7 \mathrm{~mm}$ in length and consist of 934 A-scans each. The GCC scanning protocol displays the average total, superior and inferior GCC thickness in an image display (Figure 2).

For standardisation, the clinical equipment and environment (lighting, humidity and participant proximity to the air conditioning vent) were kept as constant as possible throughout the data collection period. Moreover, the subjective refraction, corneal power measurement and OCT scans were performed by one examiner each to ensure standardisation of the data collection procedures and prevent inter-examiner variability affecting measurements. Three consecutive measurements for corneal power, RNFL and GCC thickness were recorded and the averages computed.

Data were captured and analysed with the Statistical Package for Social Sciences (SPSS) version 24 using descriptive and inferential statistics. Data are presented as means \pm standard deviations. The Shapiro-Wilk's test and graphical inspection of histograms were used to assess normality of the data. The independent sample $t$-test was used to compare RNFL and 


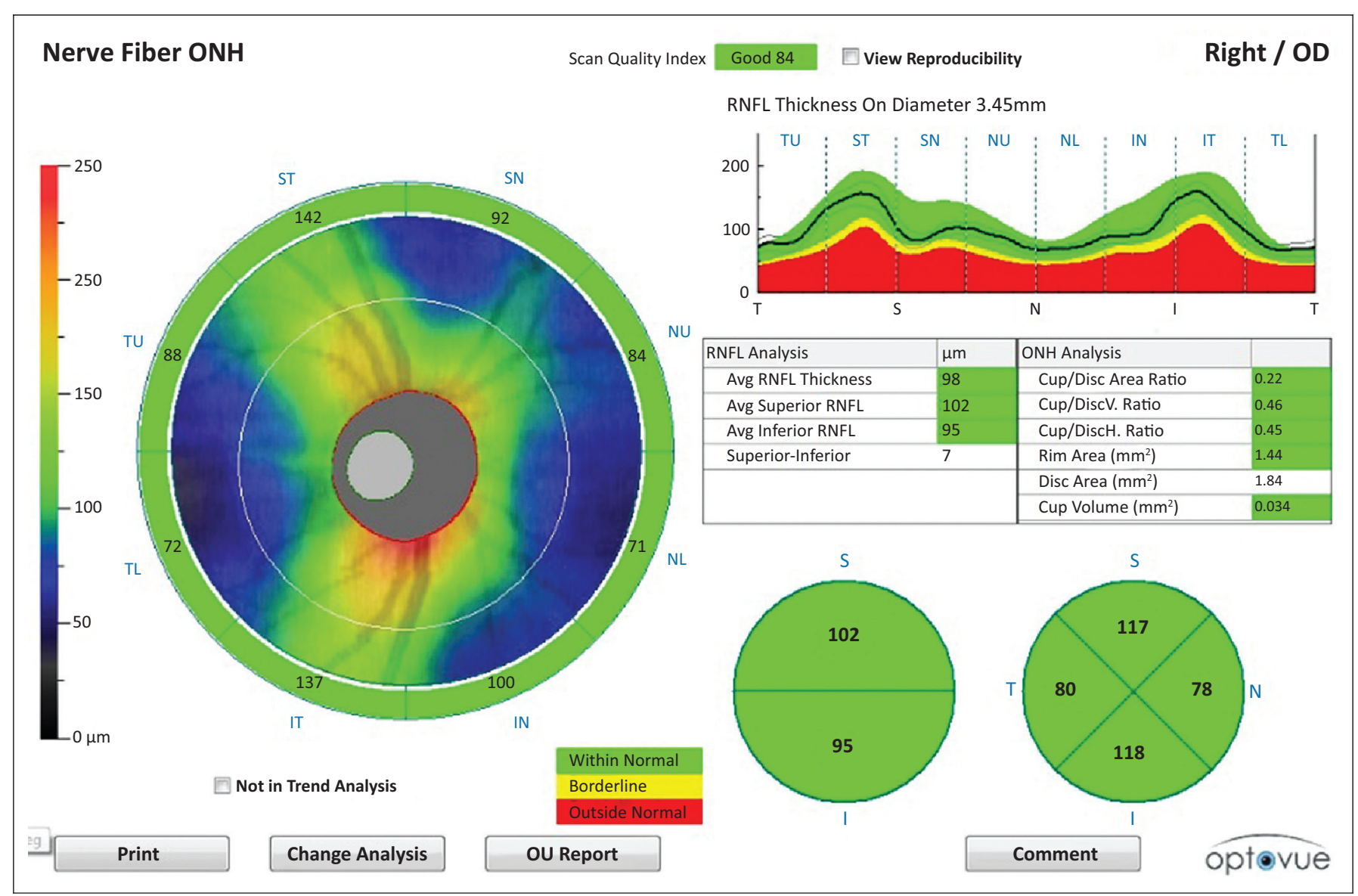

Source: Modified screen shot of the output screen with the iVue-100 OCT

FIGURE 1: A retinal nerve fibre layer map showing the average global and quadrant retinal nerve fibre layer thicknesses.

GCC thickness between the keratoconus and control groups. A probability $(p)$ value $\leq 0.05$ was considered as statistically significant.

\section{Ethical considerations}

The study (reference number BE415/16) was approved by the Biomedical Research and Ethics Committee at UKZN. Written informed consent was obtained from all participants after explaining the nature of the study and associated procedures. All ethical guidelines were adhered to during the study. After obtaining ethical approval, a pilot study was conducted to standardise the data collection procedure.

\section{Results}

The study sample consisted of 56 participants, which included 28 each in the keratoconus and control groups. There was an equal distribution of male $(n=14)$ and female $(n=14)$ participants within each group. The sample consisted of majority black people $(n=34)$ followed by Indian $(n=18)$ and mixed race $(n=4)$ participants. Ocular variables from 19 right eyes and 24 left eyes each from the keratoconus and control groups were used in the analysis for this study. Table 1 shows the demographic and ocular characteristics of the sample. There was no significant difference in mean age between the keratoconus (24.29 \pm 5.02 years) and control ( $24.11 \pm 4.67$ years) groups ( $p=0.891$ ). However, participants in the keratoconus group were significantly more myopic and had poorer best-corrected LogMAR distance visual acuity, steeper corneal powers (along the two principal meridians) as well as lower IOP measurements in both the right and left eyes $(p \leq 0.016)$.

The means and standard deviations for global RNFL thickness and the RNFL thickness in each quadrant for both eyes in the keratoconus and control groups are presented in Table 2. In the right eye, the superior quadrant was thickest followed by the inferior, nasal and temporal quadrants in both the keratoconus and control groups. Although the temporal quadrant was thinnest in both groups for the left eye, the inferior quadrant recorded the highest value $(124 \mu \mathrm{m})$ in the keratoconus group. For the control group, the superior and inferior quadrants were thickest and had an equal measurement of $132 \mu \mathrm{m}$ (Table 2).

For both the right and left eyes, the mean thickness of the nasal quadrant was almost identical in the keratoconus and control groups (Table 2). With the exception of the nasal quadrant, the control group had higher mean RNFL thickness measurements than the keratoconus group for all quadrants. However, only the temporal quadrant in the right eye and superior quadrants in both eyes, with thickness differences of $7 \mu \mathrm{m}$ and $13 \mu \mathrm{m}$ respectively, were statistically significant $(p \leq 0.036)$. In the inferior quadrant of both the right and left eyes, an insignificant thickness difference of $8 \mu \mathrm{m}$ was noted between the control and keratoconus groups (Table 2). 


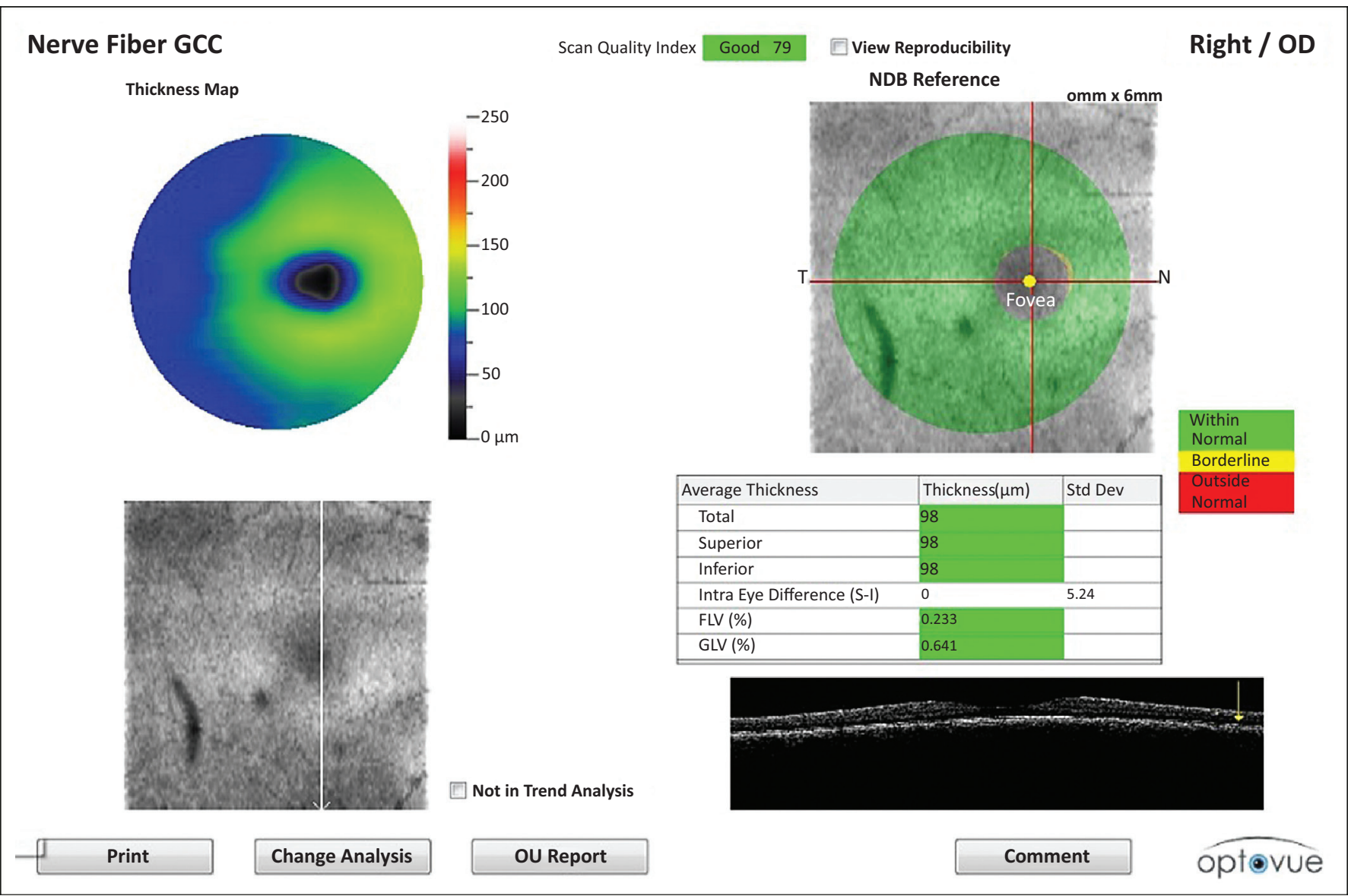

Source: Modified screen shot of the output screen with the iVue-100 OCT

FIGURE 2: A ganglion cell complex map showing the average total, superior and inferior ganglion cell complex thicknesses.

TABLE 1: Means and standard deviations for age and ocular characteristics of the keratoconus $(n=28)$ and control $(n=28)$ groups.

\begin{tabular}{llcl}
\hline Age and ocular characteristics & Keratoconus & Control & $p \dagger$ \\
\hline Age (years) & $24.29 \pm 5.02$ & $24.11 \pm 4.67$ & 0.891 \\
Right eye spherical equivalent (D) & $-5.87 \pm 4.81$ & $-0.73 \pm 1.60$ & $0.002 \ddagger$ \\
Left eye spherical equivalent (D) & $-8.51 \pm 12.39$ & $-0.70 \pm 1.35$ & $0.016 \ddagger$ \\
Right eye visual acuity (LogMAR) & $0.427 \pm 0.28$ & $-0.046 \pm 0.11$ & $<0.001 \ddagger$ \\
Left eye visual acuity (LogMAR) & $0.432 \pm 0.24$ & $-0.069 \pm 0.13$ & $<0.001 \ddagger$ \\
Right eye corneal power: K1 (D) & $49.52 \pm 4.82$ & $42.82 \pm 1.03$ & $<0.001 \ddagger$ \\
Right eye corneal power: K2 (D) & $52.72 \pm 5.88$ & $43.38 \pm 1.31$ & $<0.001 \ddagger$ \\
Left eye corneal power: K1 (D) & $50.65 \pm 6.21$ & $42.57 \pm 1.35$ & $<0.001 \ddagger$ \\
Left eye corneal power: K2 (D) & $55.27 \pm 8.77$ & $43.36 \pm 1.59$ & $<0.001 \ddagger$ \\
Right eye IOP (mmHg) & $12.20 \pm 3.40$ & $16.29 \pm 2.15$ & $<0.001 \ddagger$ \\
Left eye IOP (mmHg) & $11.35 \pm 3.82$ & $15.63 \pm 2.30$ & $<0.001 \ddagger$ \\
\hline
\end{tabular}

IOP, intraocular pressure.

$\dagger, t$-test; $\ddagger$, statistically significant.

In both eyes, the global RNFL thickness was $5 \mu \mathrm{m}-7 \mu \mathrm{m}$ higher in the control group. However, these thickness differences were not statistically significant $(p>0.05)$. For the RNFL scans, the mean scan quality index was significantly higher in the control group for both the right (77 vs. 45 , $p<0.001)$ and left (74 vs. $45, p<0.001)$ eyes.

Figure 3 shows the mean GCC thickness for the keratoconus and control groups for the superior and inferior GCC areas of the right and left eyes. Overall, the mean GCC thickness was $3 \mu \mathrm{m}-6 \mu \mathrm{m}$ higher in the control group with only the superior areas achieving statistical significance in both eyes
TABLE 2: Means and standard deviations for retinal nerve fibre layer thickness $(\mu \mathrm{m})$ in the keratoconus and control groups stratified for the right and left eyes.

\begin{tabular}{|c|c|c|c|c|c|c|}
\hline \multirow{2}{*}{$\begin{array}{l}\text { RNFL } \\
\text { variable }\end{array}$} & \multicolumn{2}{|c|}{ Right eye } & \multirow[t]{2}{*}{$p_{\dagger}^{\dagger}$} & \multicolumn{2}{|c|}{ Left eye } & \multirow[t]{2}{*}{$p \dagger$} \\
\hline & Keratoconus & Control & & Keratoconus & Control & \\
\hline Global & $99 \pm 10$ & $106 \pm 12$ & 0.057 & $98 \pm 9$ & $103 \pm 13$ & 0.113 \\
\hline Superior & $122 \pm 15$ & $135 \pm 15$ & $0.015 \$$ & $119 \pm 12$ & $132 \pm 18$ & $0.008 \$$ \\
\hline Inferior & $121 \pm 19$ & $129 \pm 16$ & 0.143 & $124 \pm 15$ & $132 \pm 16$ & 0.089 \\
\hline Nasal & $81 \pm 13$ & $80 \pm 12$ & 0.867 & $78 \pm 11$ & $78 \pm 16$ & 0.918 \\
\hline Temporal & $72 \pm 9$ & $79 \pm 9$ & $0.036 \$$ & $72 \pm 10$ & $73 \pm 9$ & 0.649 \\
\hline
\end{tabular}

RNFL, retinal nerve fibre layer.

$\dagger, t$-test; $\ddagger$, statistically significant.

$(p \leq 0.029)$. The total GGC thickness was also higher in the control group than in the keratoconus group for both the right $(99 \mu \mathrm{m}$ vs. $93 \mu \mathrm{m}, p=0.029)$ and left (97 $\mu \mathrm{m}$ vs. $93 \mu \mathrm{m}$, $p=0.051)$ eyes. For the GCC scans, the mean scan quality index was significantly higher in the control group for both the right (82 vs. $55, p<0.001)$ and left (76 vs. $52, p<0.001)$ eyes.

\section{Discussion}

In the present study, RNFL and GCC measurements were compared in a group of keratoconus and control participants accessing the UKZN optometry clinic. The results showed that both RNFL and GCC measurements were comparable between the keratoconus and control groups. The control participants had slightly higher global RNFL thickness measurements than the keratoconus participants $(106 \mu \mathrm{m}$ vs. $99 \mu \mathrm{m}$ and $103 \mu \mathrm{m}$ vs. $98 \mu \mathrm{m}$ for the right and left eyes respectively), but these 


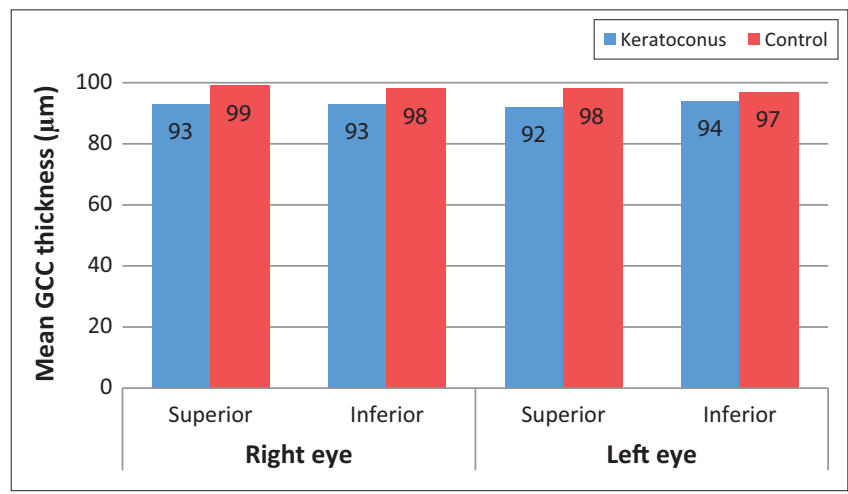

GCC, ganglion cell complex.

FIGURE 3: Ganglion cell complex thicknesses for the superior and inferior ganglion cell complex areas in the keratoconus and control groups.

differences were not statistically significant $(p \geq 0.057)$. This finding is in agreement with previous studies that have compared mean global RNFL thickness in individuals with keratoconus and controls. ${ }^{3,35}$ Cankaya et al. ${ }^{3}$ reported insignificantly thinner global RNFL thickness measurements in individuals with keratoconus than in age- and gendermatched controls $(96.5 \mu \mathrm{m}$ vs. $99.0 \mu \mathrm{m}, p=0.43)$. Bayhan et $\mathrm{al}^{35}$ reported a similar trend with thinner global RNFL thickness measurements in individuals with keratoconus compared with control participants (109.54 $\mu \mathrm{m}$ vs. $104.44 \mu \mathrm{m}, p=0.265)$. Despite also noting thicker global RNFL thickness measurements in healthy controls $(94.7 \mu \mathrm{m})$ than individuals with keratoconus $(\leq 90.7 \mu \mathrm{m})$, Uzunel et al. ${ }^{36}$ reported that these thickness differences were statistically significant $(p<$ 0.001). Unlike in the present study, Uzunel et al. ${ }^{36}$ separated their participants with keratoconus $(n=68)$ into three grades, using the Amsler-Krumeich classification system, and analysed the different mean global RNFL measurements for each grade of keratoconus which may explain this difference.

We found that quantitative comparisons of the mean RNFL thickness in the four quadrants, between the two groups, showed a similar pattern to that of the global RNFL thickness. The mean RNFL measurements were marginally higher $(1 \mu \mathrm{m}-13 \mu \mathrm{m})$ in the control group for all quadrants with the exception of only the nasal quadrant in the left eye where the same value $(78 \mu \mathrm{m})$ was noted in the two groups. This finding is consistent with reports from previous studies, ${ }^{3,35}$ which also noted lower quadrant-specific mean RNFL thickness measurements in individuals with keratoconus when compared with healthy controls. For the superior quadrant, Bayhan et al. ${ }^{35}$ reported a $13.6 \mu \mathrm{m}$ statistically significant $(p=0.045)$ RNFL thickness difference between the keratoconus and control groups which is almost identical to the difference (13 $\mu \mathrm{m}, p \leq 0.015$ ) noted for the superior quadrant of both eyes in the present study. Bayhan et al. ${ }^{35}$ used a similar Optovue-based OCT device (RTVue-100) to measure the RNFL thickness and also included young adult participants (mean age $\leq 29.47$ years) which may explain this similarity. In the present study, the mean RNFL thickness measurements for the nasal quadrants were almost identical in the two groups. This finding is in accordance with previous studies ${ }^{3,35}$ which have reported less than $3 \mu \mathrm{m}$ nasal quadrant RNFL thickness differences between individuals with keratoconus and healthy controls.

In our study, the mean GCC thickness was slightly thinner in the keratoconus group than in the control group. For the superior GCC area in both eyes, the thickness difference between the two groups was identical $(6 \mu \mathrm{m}, p \leq 0.029)$. This finding is in agreement with Bayhan et al. ${ }^{35}$ who also noted a small yet significant superior GCC thickness difference between individuals with keratoconus and healthy controls (4.6 $\mu \mathrm{m}, p=0.018)$. Moreover, the study by Bayhan et al. ${ }^{35}$ also reported an insignificant $4 \mu \mathrm{m}$ thickness difference for the inferior GCC area which is consistent with the results ( $3 \mu \mathrm{m}-5 \mu \mathrm{m}, p>0.05)$ found in the present study. For the total GCC, the two groups showed thickness differences of $4 \mu \mathrm{m}$ and $6 \mu \mathrm{m}$ for the right and left eyes respectively, which is in agreement with a previous study that reported a $5.78 \mu \mathrm{m}$ thickness difference. ${ }^{35}$

Overall, the mean global RNFL thickness, RNFL thickness in each quadrant and GCC thicknesses were slightly lower in the keratoconus group than in the control group. The extent of these thickness differences ranged from $1 \mu \mathrm{m}$ to $13 \mu \mathrm{m}$. Previous studies have reported that thicker RNFL measurements are not associated with visual field anomalies. ${ }^{37,38,39}$ Consequently, it is speculated that substantial retinal ganglion cell loss and associated RNFL thinning is necessary before a functional visual field loss is detected. ${ }^{39,40,41,42}$ A recent study ${ }^{42}$ reported that at least $8.4 \%$ of global RNFL thinning is needed to detect functional loss when evaluated with visual field assessments. A previous study, ${ }^{41}$ which measured RNFL thickness in 72 normal and 40 glaucomatous participants, reported that a $17 \%$ decrease in global RNFL thickness is necessary to detect a functional visual field loss. Moreover, these researchers ${ }^{41}$ highlighted $75.3 \mu \mathrm{m}$ as the 'tipping point' value at which visual field loss can be detected clinically. In the present study, the mean global RNFL thickness values for both the keratoconus and control groups were higher than $75.3 \mu \mathrm{m}$. In addition, the global RNFL thickness difference between the two groups is equivalent to only $6 \%$ and can therefore be regarded as clinically insignificant as they are lower than the values associated with functional losses. ${ }^{41,42}$

The exact reason for the marginal differences in RNFL and GCC thickness between the keratoconus and control groups is unknown. However, it may be related to corneal geometric and biomechanical variables including central corneal thickness (CCT) and corneal hysteresis $(\mathrm{CH})$ respectively. It is well known that keratoconus is associated with central corneal thinning and reduced CCT measurements. ${ }^{27}$ The Ocular Hypertension Treatment Study (OHTS) noted that thinner CCT measurements are a risk factor for the development of primary open-angle glaucoma. ${ }^{43}$ Moreover, previous studies have reported that eyes with thinner CCT measurements have larger ${ }^{44,45}$ and deeper optic cups. ${ }^{45}$ 
Corneal hysteresis describes the viscoelastic characteristic of the cornea ${ }^{4}$ and has been shown to vary in eyes with different ocular diseases. Research studies have consistently reported that individuals with glaucoma have significantly lower $\mathrm{CH}$ measurements than healthy controls. ${ }^{44,46}$ However, contradictory findings have been reported for $\mathrm{CH}$ measurements in individuals with keratoconus. Some studies have reported that mean $\mathrm{CH}$ measurements are significantly lower in individuals with keratoconus than normal individuals. ${ }^{28,47}$ However, Cohen and Myers ${ }^{4}$ reported that individuals with keratoconus, who either had glaucoma or were glaucoma suspects, had similar mean $\mathrm{CH}$ measurements to healthy controls. Shah et al. ${ }^{48}$ also noted that mean $\mathrm{CH}$ measurements were similar and had considerable overlap between healthy controls and individuals with mild keratoconus. There is no correlation between $\mathrm{CH}$ and RNFL or GCC thickness. ${ }^{46,49}$ Moreover, Insull et al. ${ }^{44}$ suggested that $\mathrm{CH}$ does not affect the optic disc size as it is not related to the optic disc area. It is possible that the lack of association between $\mathrm{CH}$ and RNFL, GCC as well as optic disc size may account for the similar RNFL and GCC thicknesses noted between the two groups in this study. Thus, we speculate that the $\mathrm{CH}$ measurements in the two groups were relatively similar which may explain the lack of clinically significant thickness differences between the two groups. This explanation is reasonable considering that even though there were obvious CCT differences between participants in the keratoconus and control groups, none of the participants in these groups had glaucoma and/or were suspected of having glaucoma. However, we suggest that future studies compare $\mathrm{CH}$ measurements among healthy controls, individuals with keratoconus as well as individuals with keratoconus and concurrent glaucoma to validate this speculation.

In our study, the mean global RNFL for the control group which consisted of normal healthy participants was $\sim 105 \mu \mathrm{m}$. This value is comparable to mean RNFL thickness values (range $105.5 \mu \mathrm{m}-107.7 \mu \mathrm{m}$ ), also measured with OCT devices, reported in studies involving healthy Indian, ${ }^{50,51}$ Korean $^{52,53}$ and Turkish ${ }^{54}$ populations. Despite also using a Fourier-domain OCT device to measure the RNFL thickness, Cankaya et al. ${ }^{3}$ reported a slightly lower global RNFL thickness value $(99 \mu \mathrm{m})$ which may be attributed to the inclusion of slightly older participants (mean age 31.5 years) and a larger sample size $(n=74)$ in their control group. Nevertheless, such comparisons across studies should be interpreted with caution as RNFL thickness varies with several ocular and demographic factors..$^{51,55}$

Few studies have measured RNFL thickness in individuals with keratoconus and these studies have reported conflicting results. The mean global RNFL thickness in the keratoconus group $(\sim 99 \mu \mathrm{m})$ in the present study is comparable to the mean value $(97 \mu \mathrm{m})$ reported by Cankaya et al. ${ }^{3}$ in individuals with keratoconus. In contrast, Bayhan et al. ${ }^{35}$ reported a higher mean global RNFL thickness $(104.44 \mu \mathrm{m})$. It is likely that differences in the study methodologies specifically related to the different OCT devices used to measure RNFL thickness are accountable for the variations observed. To this extent, researchers have cautioned against comparing RNFL thickness values across studies as variations have been observed even when the RNFL is measured on the same individuals with different OCT devices. ${ }^{26,56}$

It was found that, as expected, the keratoconus group was significantly more myopic and had steeper corneal power measurements as has been reported previously. ${ }^{28,29}$ In OCT devices, the scan quality index/signal strength associated with a scan is an indication of the quality of the scan. Thus, the scan quality index may influence the validity of the measurements obtained with the OCT devices. ${ }^{57,58}$ In the present study, the mean scan quality indices for the RNFL and GCC scans were higher than the minimum value (27) recommended by the manufacturer in both the keratoconus and control groups. However, the mean scan quality indices were significantly lower in the keratoconus group as has been noted in previous studies. ${ }^{59,60}$ The scan quality index may be affected by refractive changes. ${ }^{60,61}$ Consequently, the higher spherical equivalent refractive error associated with the keratoconus group is the likely explanation for this finding. Moreover, patients with keratoconus have reduced clarity of the optical media which may have also contributed to the lower scan quality indices noted in this group. ${ }^{4}$

Strengths of the present study include the use of standardised data collection measurement protocols for the subjective refraction, corneal power measurement and OCT scans. Moreover, the study used a high-resolution Fourier-domain OCT device to record several OCT measurements for each eye. Limitations of this study included the exclusion of participants with advanced keratoconus owing to reduced optical media and a relatively small sample size even though the keratoconus and control groups were matched for demographic characteristics. Therefore, it is recommended that future studies include a larger sample size and participants with different stages of keratoconus to investigate RNFL and GCC measurements in the different stages of keratoconus. In addition, the influence of keratoconus on retinal thickness measurements for all layers should be investigated, as this study only focused on the RNFL and GCC thicknesses.

\section{Conclusion}

The results of this study are important and may help in diagnosing and monitoring glaucoma in individuals with keratoconus. Evaluation of the GCC is important as it has good sensitivity in differentiating between healthy and glaucomatous eyes which may be a consequence of GCC changes occurring early in glaucoma. ${ }^{62,63}$ Thus, it is proposed that evaluation of the GCC in addition to the RNFL may be more important especially in individuals with keratoconus where other clinical tests (tonometry, optic nerve head assessment and visual fields) necessary for evaluation of glaucoma may be unreliable. The study concludes that keratoconus is associated with RNFL and GCC thickness changes that are not clinically significant. Therefore, abnormally reduced RNFL and GCC thickness in patients 
with keratoconus warrants further investigation for retinal and optic nerve head pathologies specifically glaucoma.

\section{Acknowledgements}

The authors acknowledge Professor C.P. Brown from the Florida Agricultural and Mechanical University for assistance with statistical analysis. At the time of the study, Professor Brown was assisting with statistical guidance at the UKZN.

\section{Competing interests}

The authors declare that they have no financial or personal relationships that may have inappropriately influenced them in writing this article.

\section{Authors' contributions}

D.S. was the study leader. N.G. and N.R. provided feedback on the study design. P.C., N.M., M.M., K.M.E., D.S. and N.W. performed the data collection. N.R. and N.G. wrote the manuscript.

\section{References}

1. Rabinowitz YS. Keratoconus. Surv Ophthalmol. 1998;42:297-319. https://doi. org/10.1016/S0039-6257(97)00119-7

2. Kanski JJ. Clinical ophthalmology: A systematic approach. 6th ed. London: Butterworth-Heinmann; 2007

3. Cankaya AB, Beyazyildiz E, lleri D, Yilmazbas P. Optic disc and retinal nerve fibe layer parameters of eyes with keratoconus. Ophthalmic Surg. 2012;43:401-407. layer parameters of eyes with keratoconus. Ophthat
https://doi.org/10.3928/15428877-20120531-01

4. Cohen EJ, Myers JS. Keratoconus and normal-tension glaucoma: A study of the possible association with abnormal biomechanical properties as measured by possible association with abnormal biomechanical properties as measured by
corneal hysteresis. Cornea. 2010;29:955-970. https://doi.org/10.1097/ICO.0b01 3e3181ca363c

5. Francis BA, Hsieh A, Lai M, et al. Effects of corneal thickness, corneal curvature, and intraocular pressure level on Goldmann applanation tonometry and dynamic contour tonometry. Ophthalmology. 2007;114:20-26. https://doi.org/10.1016/ j.ophtha.2006.06.047

6. Tham YC, Li X, Wong TY, Quigley HA, Aung T, Cheng CY. Global prevalence of glaucoma and projections of glaucoma burden through 2040: A systematic review and meta-analysis. Ophthalmology. 2014;121:2081-2090. https://doi.org/ 10.1016/j.ophtha.2014.05.013

7. Shih CY, Graff Zivin JS, Trokel SL, Tsai JC. Clinical significance of central corneal thickness in the management of glaucoma. Arch Ophthalmol. 2004;122 1270-1275. https://doi.org/10.1001/archopht.122.9.1270

8. Thomas R, Korah S, Muliyil J. The role of central corneal thickness in the diagnosis of glaucoma. Indian J Ophthalmol. 2000;48:107-111.

9. Stamper RL, Lieberman MF, Drake MV. Becker-Shaffer's diagnosis and therapy of the glaucomas. 8th ed. Edinburgh: Mosby Elsevier, 2009; p. 144-145.

10. Townsend KA, Wollstein G, Schuman JS. Imaging of the retinal nerve fiber layer for glaucoma. Br J Ophthalmol. 2009;93:139-143. https://doi.org/10.1136/bjo. 2008.145540

11. Huang D, Swanson EA, Lin CP, et al. Optical coherence tomography. Science. 1991;254:1178-1181. https://doi.org/10.1126/science.1957169

12. Tanigawa J, Barlis P, Di Mario C. Intravascular optical coherence tomography: Optimisation of image acquisition and quantitative assessment of stent strut apposition. Eurolntervention. 2007;3:128-136.

13. Wojtkowski M, Kaluzny B, Zawadzki RJ. New directions in ophthalmic optical coherence tomography. Optom Vis Sci. 2012;89:524-542. https://doi.org/ coherence tomography. Optom
$10.1097 / O P X .0 b 013 \mathrm{e} 31824$ eecb2

14. Yabushita H, Bourma BE, Houser SL, et al. Characterization of human atherosclerosis by optical coherence tomography. Circulation. 2002;106: 1640-1645. https://doi.org/10.1161/01.CIR.0000029927.92825.F6

15. Hrynchak P, Simpson T. Optical coherence tomography: An introduction to the technique and its use. Optom Vis Sci. 2000;77:347-356. https://doi.org/ 10.1097/00006324-200007000-00009

16. Srinivasen VJ, Wojtkowski M, Witkin AJ, et al. High-definition and 3-dimensional imaging of macular pathologies with high-speed ultrahigh-resolution optical coherence tomography. Ophthalmology. 2006;113:2054.e1-2054.14.

17. Hong JP, Kim T, Chung JL, Huang D, Cho HS, Kim EK. Analysis of deposit depth and morphology in granular corneal dystrophy type 2 using Fourier domain optical coherence tomography. Cornea. 2011;30:729-738. https://doi.org/10.1097/ ICO.0b013e3182000933
18. Christopoulos V, Kagemann L, Wollstein G, et al. In vivo corneal high-speed, ultrahigh-resolution optical coherence tomography. Arch Ophthalmol. 2007:125:10271035. https://doi.org/10.1001/archopht.125.8.1027

19. Quek DT, Narayanaswamy AK, Tun TA, et al. Comparison of two spectral domain optical coherence tomography devices for angle-closure assessment. Invest Ophthalmol Vis Sci. 2012;53:5131-5136. https://doi.org/10.1167/iovs.12-10132

20. Asrani S, Surunic M, Santiago C, Izatt J. Detailed visualization of the anterior segment using Fourier-domain optical coherence tomography. Arch Ophthalmol. 2008;126:765-771. https://doi.org/10.1001/archopht.126.6.765

21. Sharma R, Sharma A, Arora T, et al. Application of anterior segment optical coherence tomography in glaucoma. Surv Ophthalmol. 2014;59:311-327. https:// doi.org/10.1016/j.survophthal.2013.06.005

22. Radhakrishnan S, Yarovoy D. Development in anterior segment imaging for glaucoma. Curr Opin Ophthalmol. 2014;25(2):98-103. https://doi.org/10.1097/ ICU.0000000000000026

23. Reibaldi M, Uva MG, Avitabile T, et al. Intrasession reproducibility of RNFL thickness measurements using SD-OCT in eyes with keratoconus. Ophthalmic Surg Lasers Imaging. 2012;43:83-89. https://doi.org/10.3928/15428877-20121001-04

24. Reibaldi M, Reibaldi A, Avitabile T, et al. Reproducibility of retinal nerve fiber layer thickness measurements using spectral domain optical coherence tomography in eyes with keratoconus. Invest Ophthalmol Vis Sci. 2011;52:3664.

25. Garas A, Tóth M, Vargha P, Holló G. Comparison of repeatability of retinal nerve fiber layer thickness measurement made using the RTVue Fourier-domain optical coherence tomograph and the GDx scanning laser polarimeter with variable or enhanced corneal compensation. J Glaucoma. 2010;19(6):412-417. https://doi. org/10.1097/IJG.0b013e3181bdb549

26. Vizzeri G, Weinreb RN, Gonzalez-Garcia AO, et al. Agreement between spectraldomain and time-domain OCT for measuring RNFL thickness. Br J Ophthalmol. 2009;93:775-781. https://doi.org/10.1136/bjo.2008.150698

27. Emre S, Doganay S, Yologlu S. Evaluation of anterior segment parameters in keratoconus eyes measured with the Pentacam system. J Cataract Refract Surg. 2007;33:1708-1712. https://doi.org/10.1016/j.jcrs.2007.06.020

28. Fontes BM, Ambrósio R, Jardim D, Verlarde GC, Nosé W. Corneal biomechanical metrics and anterior segment parameters in mild keratoconus. Ophthalmology. 2010;117:673-679. https://doi.org/10.1016/j.ophtha.2009.09.023

29. Piñero DP, Alió JL, Alesón A, Vergara ME, Miranda M. Corneal volume, pachymetry, and correlation of anterior and posterior corneal shape in subclinical and different stages of clinical keratoconus. J Cataract Refract Surg. 2010;36:814-825. https:// doi.org/10.1016/j.jcrs.2009.11.012

30. Hougaard JL, Ostenfeld C, Heijl A, Bengtsson B. Modelling the normal retinal nerve fibre layer thickness as measured by Stratus optical coherence tomography. Graefe Arch Clin Exp Ophthalmol. 2006;12:1607-1614. https://doi.org/10.1007/ Graefe Arch Clin Exp

31. Alasil T, Wang K, Keane PA, et al. Analysis of normal retinal fiber layer thickness by age, sex, and race using spectral domain optical coherence tomography.
J Glaucoma. 2013;22:532-541. https://doi.org/10.1097/IJG.0b013e318255bb4a

32. Stephens GL. Correction with single-vision spectacle lenses. In: Benjamin WJ, editor. Borish's clinical refraction. 2nd ed. China: Butterworth-Heinemann, 2006; p. 1026-1098.

33. Mao X, Savini G, Zhuo Z, et al. Repeatability, reproducibility, and agreement of corneal power measurements obtained with a new corneal topographer. J Cataract Refract Surg. 2013;39:1561-1569. https://doi.org/10.1016/j.jcrs.2013. 04.029

34. Optovue Inc. iVue 100 user's manual version 1.9. Fremont, CA: Optovue Inc.; 2011.

35. Bayhan SA, Bayhan HA, Gurdal C. Evaluation of the retinal nerve fiber layer and ganglion cell complex thickness with keratoconus. Turkinje Klinikleri J Ophthalmol. 2014;23:207-211

36. Uzunel UD, Küsbeci T, Yüksel B. Does the stage of keratoconus affect optical coherence tomography measurements? Semin Ophthalmol [serial online]. 2016 [cited 2017 Jun 12]. Available from: https://www.researchgate.net/profile/Umut Uzunel/publication/304706867 Does the Stage of Keratoconus Affect Optical_Coherence_Tomography_Measurements/links/57a8637508aed 6703f4caf1.pdf

37. Ajtony C, Balla Z, Somoskeoy S, Kovacs B. Relationship between visual field sensitivity and retinal nerve fiber layer thickness as measured by optical coherence tomography. Invest Ophthalmol Vis Sci. 2007;48:258-263. https://doi. org/10.1167/iovs.06-0410

38. Schlottmann PG, De Cilla S, Greenfield DS, Caprioli J, Garway-Heath DF Relationship between visual field sensitivity and retinal nerve fiber layer thickness as measured by scanning laser polarimetry. Invest Ophthalmol Vis Sci. 2004;45:1823-1829. https://doi.org/10.1167/iovs.03-0692

39. Hood DC, Kardon RH. A framework for comparing structural and functional measures of glaucomatous damage. Prog Retin Eye Res. 2007;26:688-710. https://doi.org/10.1016/j.preteyeres.2007.08.001

40. Kerrigan-Baumrind LA, Quigley HA, Pease ME, Kerrigan DF, Mitchell RS. Number of ganglion cells in glaucoma eyes compared with threshold visual field tests in the same persons. Invest Ophthalmol Vis Sci. 2000;41:741-748.

41. Wollstein G, Kagemann L, Bilonick RA, et al. Retinal nerve fibre layer and visual function loss in glaucoma: The tipping point. Br J Ophthalmol. 2012;96:47-52. https://doi.org/10.1136/bjo.2010.196907

42. Alasil TA, Wang K, Yu F, et al. Correlation of retinal nerve fiber layer thickness and visual fields in glaucoma: A broken stick model. Am J Ophthalmol. 2014;157: 953-959. https://doi.org/10.1016/j.ajo.2014.01.014 
43. Gordon MO, Beiser JA, Brandt JD, et al. The Ocular Hypertension Treatment Study: Baseline factors that predict the onset of primary open-angle glaucoma. Arch Ophthalmol. 2002;120:714-720. https://doi.org/10.1001/archopht.120.6.714

44. Insull E, Nicholas S, Ang GS, Poostchi A, Chan K, Wells A. Optic disc area and correlation with central corneal thickness, corneal hysteresis and ocular pulse amplitude in glaucoma patients and controls. Clin Experiment Ophthalmol. 2010;38:839-844. https://doi.org/10.1111/j.1442-9071.2010.02373.x

45. Gunvant P, Porsia L, Watkins RJ, Bayliss-Brown H, Broadway DC. Relationships between central corneal thickness and optic disc topography in eyes with glaucoma, suspicion of glaucoma, or ocular hypertension. Clin Ophthalmol. 2008;2:591-599. https://doi.org/10.2147/OPTH.S2814

46. Mansouri K, Leite MT, Weinreb RN, Tafreshi A, Zangwill L, Medeiros FA. Association between corneal biomechanical properties and glaucoma severity. Am J Ophthalmol. 2012;153(3):419-427.e1. https://doi.org/10.1016/j.ajo.2011.08.022

47. Ortiz D, Piñero D, Shabayek MH, Arnalich-Montiel F, Alió JL. Corneal biomechanical properties in normal, post-laser in situ keratomileusis and keratoconic eyes. J Cataract Refract Surg. 2007;33:1371-1375. https://doi.org/10.1016/j.jcrs 2007.04.021

48. Shah S, Laiquzzaman M, Bhojwani R, Mantry S, Cunliffe I. Assessment of the biomechanical properties of the corneal with the Ocular Response Analyzer in normal and keratoconic eyes. Invest Ophthalmol Vis Sci. 2007;48:3026-3031. https://doi.org/10.1167/iovs.04-0694

49. Chang PY, Chang SW. Corneal biomechanics, optic disc morphology, and macular ganglion cell complex in myopia. J Glaucoma. 2013;22:358-362. https://doi. org/10.1097/IJG.0b013e3182447a17

50. Pawar N, Maheshwari D, Ravindran M, Ramakrishnan R. Retinal nerve fiber layer thickness in normal Indian pediatric population measured with optical coherence tomography. Indian J Ophthalmol. 2014;62:412-418. https://doi.org/10.4103/ 0301-4738.121185

51. Budenz DL, Anderson DR, Varma R, et al. Determinants of normal retinal nerve fiber layer thickness measured by Stratus OCT. Ophthalmology. 2007;114 1046-1052. https://doi.org/10.1016/j.ophtha.2006.08.046

52. Kim MJ, Lee EJ, Kim TW. Peripapillary retinal nerve fiber layer thickness profile in subjects with myopia measured using the Stratus optical coherence tomography. Br J Ophthalmol. 2010;94:115-120. https://doi.org/10.1136/bjo. 2009.162206
53. Ahn HC, Son HW, Kim JS, Lee JH. Quantitative analysis of retinal nerve fiber layer thickness of normal children and adolescents. Kor J Ophthalmol. 2005;19: 195-200. https://doi.org/10.3341/kjo.2005.19.3.195

54. Turk A, Ceylan OM, Arici C, et al. Evaluation of the nerve fiber layer and macula in the eyes of healthy children using spectral-domain optical coherence tomography. Am J Ophthalmol. 2012;153:552-559. https://doi.org/10.1016/j.ajo.2011.08.026

55. Mashige KP, Oduntan OA. Retinal nerve fibre layer thickness values and their associations with ocular and systemic parameters in Black South Africans. Afr Health Sci. 2016;16:1188-1194. https://doi.org/10.4314/ahs.v16i4.39

56. Sung KR, Kim DY, Park SB, Kook MS. Comparison of retinal nerve fiber laye thickness measured by Cirrus HD and Stratus optical coherence tomography. Ophthalmology. 2009;116:1264-1270. https://doi.org/10.1016/j.ophtha.2008. 12.045

57. Wu Z, Huang J, Dustin L, Sadda SR. Signal strength is an important determinant of accuracy of nerve fiber layer thickness measurement by optical coherence tomography. J Glaucoma. 2009;18:213-216. https://doi.org/10.1097/IJG.0b013e 31817eee20

58. Cheung CYL, Leung CKS, Lin D, Pang C-P, Lam DSC. Relationship between retinal nerve fiber layer measurements and signal strength in optical coherence tomography. Ophthalmology. 2008;115:1347-1351. https://doi.org/10.1016/ j.ophtha.2007.11.027

59. Uzunel UD, Kusbeci T, Yuce B, Yüksel B. Effects of rigid contact lenses on optical coherence tomographic parameters in eyes with keratoconus. Clin Exp Optom. 2015;98:319-322. https://doi.org/10.1111/cxo.12287

60. Salchow DJ, Hwang AM, Li FY, Dziura J. Effect of contact lens power on optical coherence tomography of the retinal nerve fiber layer. Invest Ophthalmol Vis Sci. 2011;52:1650-1654. https://doi.org/10.1167/iovs.10-6118

61. Lee J, Kim NR, Kim H, et al. Negative refraction power causes underestimation of peripapillary retinal nerve fibre layer thickness in spectral-domain optical coherence tomography. Br J Ophthalmol. 2011;95:1284-1289. https://doi.org/ 10.1136/bjo.2010.186536

62. Oli A, Joshi D. Can ganglion cell complex assessment on cirrus HD OCT aid in detection of early glaucoma? Saudi J Ophthalmol. 2015;29:201-204. https://doi. org/10.1016/j.sjopt.2015.02.007

63. Takayama K, Hangai M, Durbin M, et al. A novel method to detect local ganglion cell loss in early glaucoma using spectral-domain optical coherence. Invest Ophthalmol Vis Sci. 2012;53:6904-6913. https://doi.org/10.1167/iovs.12-10210 